\title{
Infrared properties of Active OB stars in the Magellanic Clouds from the Spitzer SAGE Survey
}

\author{
A.Z. Bonanos ${ }^{1}$, D.J. Lennon, D.L. Massa, M. Sewilo, F. Koehlinger, \\ N. Panagia, J.Th. van Loon, C.J. Evans, L.J. Smith, M. Meixner, \\ K. Gordon and the SAGE teams \\ ${ }^{1}$ National Observatory of Athens, IAA, \\ I. Metaxa \& Vas. Pavlou Street, Palaia Penteli GR-15236, Greece \\ email: bonanos@astro.noa.gr
}

\begin{abstract}
.
We present a study of the infrared properties of 4922 spectroscopically confirmed massive stars in the Large and Small Magellanic Clouds, focusing on the active OB star population. Besides OB stars, our sample includes yellow and red supergiants, Wolf-Rayet stars, Luminous Blue Variables (LBVs) and supergiant $\mathrm{B}[\mathrm{e}]$ stars. We detect a distinct Be star sequence, displaced to the red, and find a higher fraction of $\mathrm{Oe}$ and Be stars among $\mathrm{O}$ and early- $\mathrm{B}$ stars in the SMC, respectively, when compared to the LMC, and that the SMC Be stars occur at higher luminosities. We also find photometric variability among the active OB population and evidence for transitions of Be stars to B stars and vice versa. We furthermore confirm the presence of dust around all the supergiant $\mathrm{B}[\mathrm{e}]$ stars in our sample, finding the shape of their spectral energy distributions (SEDs) to be very similar, in contrast to the variety of SED shapes among the spectrally variable LBVs.
\end{abstract}

Keywords. catalogs- galaxies: individual (LMC, SMC)- infrared: stars- stars: early-type- stars: emission-line, Be- stars: massive

\section{Introduction}

The Spitzer Space Telescope Legacy Surveys SAGE ("Surveying the Agents of a Galaxy's Evolution", Meixner et al. 2006) and SAGE-SMC (Gordon et al. 2010) have for the first time made possible a comparative study of the infrared properties of massive stars at a range of metallicities, by imaging both the Large and Small Magellanic Clouds (LMC and SMC). In Bonanos et al. (2009, Paper I) and Bonanos et al. (2010, Paper II), we presented infrared properties of massive stars in the LMC and SMC, which we summarize below. The motivation was threefold: (a) to use the infrared excesses of massive stars to probe their winds, circumstellar gas and dust, (b) to provide a template for studies of other, more distant, galaxies, and (c) to investigate the dependence of the infrared properties on metallicity. Papers I and II were the first major compilations of accurate spectral types and multi-band photometry from $0.3-24 \mu \mathrm{m}$ for massive stars in any galaxy, increasing by an order of magnitude the number of massive stars for which mid-infrared photometry was available.

Infrared excess in hot massive stars is primarily due to free-free emission from their ionized, line driven, stellar winds. Panagia \& Felli (1975) and Wright \& Barlow (1975) first computed the free-free emission from ionized envelopes of hot massive stars, as a function of the mass-loss rate $(\dot{M})$ and the terminal velocity of the wind $\left(v_{\infty}\right)$. The properties of massive stars, and in particular their stellar winds (which affect their evolution) 
are expected to depend on metallicity $(Z)$. For example, Mokiem et al. (2007) found empirically that mass-loss rates scale as $\dot{M} \sim Z^{0.83 \pm 0.16}$, in good agreement with theoretical predictions (Vink et al. 2001). The expectation, therefore, is that the infrared excesses of OB stars in the SMC should be lower than in the LMC, given that $\dot{M}$ is lower in the SMC. Furthermore, there is strong evidence that the fraction of classical Be stars among B-type stars is higher at lower metallicity (Martayan et al. 2007). Grebel et al. (1992) were the first to find evidence for this, by showing that the cluster NGC 330 in the SMC has the largest fraction of Be stars of any known cluster in the Galaxy, LMC or SMC. More recent spectroscopic surveys (Martayan et al. 2010) have reinforced this result. We are also interested in quantifying the global dependence of the Be star fraction on metallicity. The incidence of Be/X-ray binaries is also much higher in the SMC than in the LMC (Liu et al. 2005), while the incidence of Wolf-Rayet (WR) stars is much lower; therefore, a comparison of infrared excesses for these objects is also of interest.

\section{Spectral type and Photometric Catalogs}

We have compiled catalogs of massive stars with known spectral types in both the LMC and SMC from the literature. We then cross-matched the stars in the SAGE and SAGE-SMC databases, after incorporating optical and near-infrared photometry from recent surveys of the Magellanic Clouds. The resulting photometric catalogs were used to study the infrared properties of the stars. The LMC spectral type catalog contains 1750 massive stars. A subset of 1268 of these are included in the photometric catalog, for which uniform photometry from $0.3-24 \mu \mathrm{m}$ in the $U B V I J H K_{s}+$ IRAC+MIPS24 bands is presented in Paper I. The SMC spectral type catalog contains 5324 massive stars; 3654 of these are included in the photometric catalog, for which uniform photometry from $0.3-24 \mu \mathrm{m}$ is presented in Paper II. All catalogs are available electronically.

\section{Active OB stars}

\subsection{O/Oe and early-B/Be stars}

In Figure 1, we plot $J_{I R S F}$ vs. $J_{I R S F}-[3.6], J_{I R S F}-[5.8]$ and $J_{I R S F}-[8.0]$ colors for the 1967 early-B stars from our SMC catalog, respectively, denoting their luminosity classes, binarity and emission line classification properties by different symbols. We compare the observed colors with colors of plane-parallel non-LTE TLUSTY stellar atmosphere models (Lanz \& Hubeny 2003, 2007) of appropriate metallicity and effective temperatures. For reference, reddening vectors and TLUSTY models reddened by $E(B-V)=0.2$ mag are also shown. We clearly detect infrared excesses from free-free emission despite not having dereddened the stars, as in the LMC. At longer wavelengths, the excess is larger because the flux due to free-free emission for optically thin winds remains essentially constant with wavelength. Fewer stars are detected at longer wavelengths because of the decreasing sensitivity of Spitzer and the overall decline of their SEDs. We find that the majority of early-B supergiants in the SMC exhibit lower infrared excesses, when compared to their counterparts in the LMC, due to their lower mass-loss rates, although certain exceptions exist and deserve further study.

The CMDs allow us to study the frequency of Oe and Be stars, given the low foreground and internal reddening for the SMC. Our SMC catalog contains 4 Oe stars among 208 O stars, of which one is bluer than the rest. There are 16 additional stars with $J_{I R S F}-$ [3.6] $>0.5 \mathrm{mag}$ and $J_{I R S F}<15 \mathrm{mag}$ (including all luminosity classes), whose spectra appear normal (although the $\mathrm{H} \alpha$ spectral region in most cases was not observed). We refer to these as "photometric Oe" stars and attribute their infrared excesses to free-free emission from a short-lived, possibly recurrent circumstellar region, whose $\mathrm{H} \alpha$ emission 
line was not detected during the spectroscopic observations either because the gas had dispersed or because the region was optically thick to $\mathrm{H} \alpha$ radiation or the observation spectral range just did not extend to $\mathrm{H} \alpha$. Given the expectation of lower $\dot{M}$ at SMC metallicity, we argue that such a region is more likely to be a transient disk rather than a wind. Assuming these are all Oe stars, we find a $10 \pm 2 \%$ fraction of Oe stars among the $\mathrm{O}$ stars in the SMC. The error in the fraction is dominated by small number statistics. In contrast, there are 4 Oe and 14 "photometric Oe" stars (with $J_{I R S F}-[3.6]>0.5 \mathrm{mag}$ and $J_{I R S F}<14.5 \mathrm{mag}$ ) out of $354 \mathrm{O}$ stars in the LMC (despite the higher $\dot{M}$ at LMC metallicity), which yields a $5 \pm 1 \%$ fraction of Oe stars among $\mathrm{O}$ stars in the LMC.

Turning to the early-B stars, the most striking feature in Figure 1 is a distinct sequence displaced by $\sim 0.8 \mathrm{mag}$ to the red. A large fraction of the stars falling on this redder sequence have Be star classifications, although not all Be stars reside there. Given that the circumstellar gas disks responsible for the emission in Be stars are known to completely vanish and reappear between spectra taken even 1 year apart (see review by Porter \& Rivinius 2003, and references therein), the double sequence reported here provides further evidence for the transient nature of the Be phenomenon. A bimodal distribution at the $L$-band was previously suggested by the study of Dougherty et al. (1994), which included a sample of 144 Galactic Be stars. Our larger Be sample, which is essentially unaffected by reddening, and the inclusion of all early-B stars, clearly confirms the bimodal distribution. It is due to the much larger number of Be stars classified in the $\mathrm{SMC}$, in comparison to the LMC, as well as the higher fraction of Be stars among early-B stars in the SMC, which is $19 \pm 1 \%$ vs. $4 \pm 1 \%$ in the LMC when considering only the spectroscopically confirmed Be stars (cf. 17\% for < 10 Myr B0-5 stars; Wisniewski et al. 2006). Excluding the targeted sample of Martayan et al. (2007a, 2007b) does not significantly bias the statistics, since the fraction only decreases to $15 \pm 1 \%$. We caution that incompleteness in our catalogs could also affect the determined fractions, if our sample turns out not to be representative of the whole population of OB stars.

We proceed to define "photometric Be" stars as early-B type stars with an intrinsic color $J_{I R S F}-[3.6]>0.5 \mathrm{mag}$, given that a circumstellar disk or envelope is required to explain such large excesses. Including these "photometric Be" stars and using the same color and magnitude cuts as for the "photometric Oe" stars above, yields fractions of Be stars among early-B stars of $27 \pm 2 \%$ for the SMC and $16 \pm 2 \%$ for the LMC (cf. $32 \%$ from young SMC clusters; Wisniewski et al. 2006). We compare our results with the fractions determined by Maeder et al. (1999) from young clusters, i.e. $39 \%$ for the SMC and $23 \%$ for the LMC, finding ours to be lower, although the sample selections were very different.

These preliminary statistics (available for the first time for Oe stars) indicate that both Oe and Be stars are twice as common in the SMC than in the LMC. We emphasize the importance of including the "photometric Be" stars, which significantly increase the frequencies of $\mathrm{Oe} / \mathrm{O}$ and $\mathrm{Be} /$ early-B stars determined and are crucial when comparing such stars in different galaxies. This novel method of confirming Oe and Be star candidates from their infrared colors or a combination of their optical and infrared colors, as recently suggested by Ita et al. (2010) is complementary to the detailed spectroscopic analyses by e.g. Negueruela et al. (2004) on individual Oe stars to understand their nature, although it is limited to galaxies with low internal reddening. We finally note that the spectral types of Oe stars in the SMC (O7.5Ve, O7Ve, O4-7Ve and O9-B0III-Ve) and the LMC (O9Ve (Fe II), O7:Ve, O8-9IIIne, O3e) are earlier than those of known Galactic Oe stars, which are all found in the O9-B0 range (Negueruela et al. 2004).

Finally, we note that the brightest Be stars in the SMC $\left(J_{I R S F} \sim 13.2 \mathrm{mag}\right)$ are brighter than the brightest Be stars in the $\mathrm{LMC}\left(J_{I R S F} \sim 13.4 \mathrm{mag}\right)$, i.e. there is a 
0.7 mag difference in absolute magnitude, given the 0.5 mag difference in the distance moduli.

\subsubsection{Supergiant $B[e]$ stars}

In the SMC photometric catalog, we have detected 7 luminous sources with colors typical of $\mathrm{sgB}[\mathrm{e}]$ stars (see Paper I for an introduction), i.e. $M_{3.6}<-8,[3.6]-[4.5]>0.7$, $J-[3.6]>2$ mag. Five of these are previously known $\mathrm{sgB}[\mathrm{e}]$ stars (with R50; B2-3[e] being the brightest in all IRAC and MIPS bands), while R4 (AzV 16) is classified as an LBV with a $\mathrm{sgB}[\mathrm{e}]$ spectral type. In addition to these, we find that 2dFS1804 (AFA3kF0/B[e]) has a very similar SED (and therefore infrared colors) to the known sgB[e] 2dFS2837 (AFA5kF0/B[e]). Evans et al. (2004) also remarked on the similarity of their spectra. We therefore confirm the supergiant nature of 2dFS1804. The similarity of the SEDs of these $\mathrm{sgB}[\mathrm{e}]$ stars, despite the various optical spectral classifications, implies that all are the same class of object. The cooler, composite spectral types indicate a lower mass and perhaps a transitional stage to or from the $\mathrm{sgB}[\mathrm{e}]$ phenomenon. The only difference we find between the $\mathrm{sgB}[\mathrm{e}]$ stars in the SMC vs. the LMC is that on average they are $\sim 1-2$ mag fainter (in absolute terms).

\subsubsection{Luminous Blue Variables}

All 3 known LBVs in the SMC: R4 (AzV 16, B0[e]LBV), R40 (AzV 415, A2Ia: LBV) and HD 5980 (WN6h;LBV binary), were detected at infrared wavelengths. R4 is the more

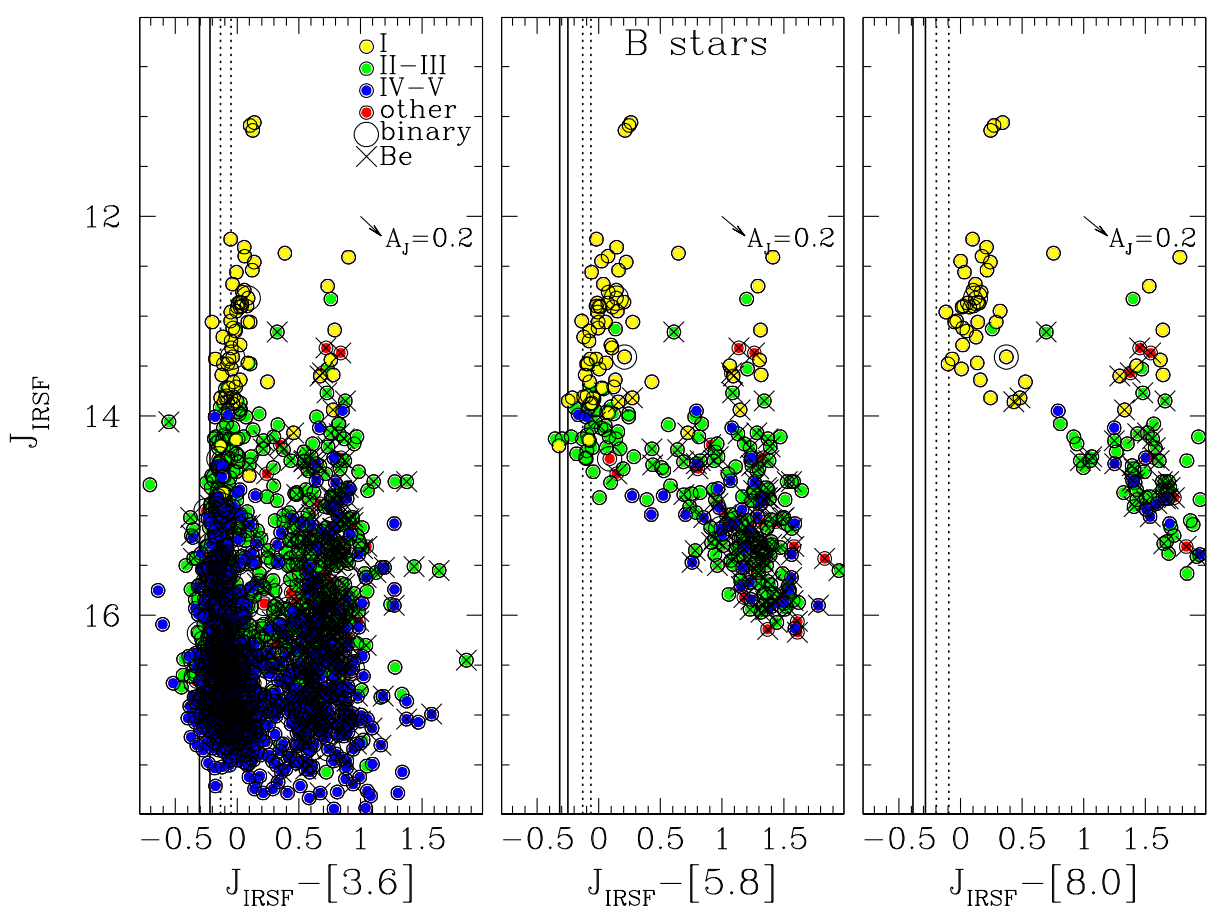

Figure 1. Infrared excesses ( $J_{I R S F}$ vs. $J_{I R S F}-[3.6], J_{I R S F}-[5.8]$ and $\left.J_{I R S F}-[8.0]\right)$ for 1967 early-B stars in the SMC. Supergiants are shown in yellow, giants in green, main-sequence stars in blue, stars with uncertain classifications ("other") in red, binaries with a large circle and Oe stars with an $\times$. The solid lines correspond to $30 \mathrm{kK}$ and $50 \mathrm{kK}$ TLUSTY models with $\log g=4.0$. A reddening vector for $E(B-V)=0.2 \mathrm{mag}$ is shown, as well as reddened TLUSTY models by this same amount (dotted lines). The more luminous stars exhibit larger infrared excesses, which increase with $\lambda$. 
reddened LBV, whereas the colors of HD 5980 (a well known eccentric eclipsing binary, see e.g. Foellmi et al. 2008) are similar to those of the LBVs in the LMC. We find their SEDs to differ, given their very different spectral types. Moreover, we find evidence for variability, which can be confirmed from existing light curves in the All Sky Automated Survey (ASAS) (Pojmanski 2002), as pointed out by Szczygiel et al. (2010), who studied the variability of the massive stars presented in Paper I in the LMC. The various SED shapes and spectral types observed depend on the time since the last outburst event and the amount of dust formed.

\section{References}

Bonanos, A. Z., Massa, D. L., Sewilo, M., et al. 2009, AJ, 138, 1003

Bonanos, A. Z., Lennon, D.J., Koehlinger, F. et al. 2010, $A J$, 140, 416

Dougherty, S. M., Waters, L. B. F. M., Burki, G., et al. 1994, A\&A, 290, 609

Evans, C. J., Lennon, D. J., Trundle, C., et al. 2004, ApJ, 607, 451

Foellmi, C., Koenigsberger, G., Georgiev, L., et al. 2008, RevMexAA, 44, 3

Gordon, K. D., Meixner, M., Blum, R., et al. 2010, $A J$, in preparation

Grebel, E. K., Richtler, T., \& de Boer, K. S. 1992, $A \xi A$, 254, L5

Ita, Y., Matsuura, M., Ishihara, D., et al. 2010a, $A \& A, 514,2$

Lanz, T. \& Hubeny, I. 2003, ApJS, 146, 417

—. 2007, ApJS, 169, 83

Liu, Q. Z., van Paradijs, J., \& van den Heuvel, E. P. J. 2005, A\& A, 442, 1135

Maeder, A., Grebel, E. K., \& Mermilliod, J. 1999, A\&A, 346, 459

Martayan, C., Floquet, M., Hubert, A. M., et al. 2007a, $A \mathscr{E} A$, 472, 577

Martayan, C., Frémat, Y., Hubert, A., et al. 2007b, A\& $A, 462,683$

Martayan, C., Baade, D., \& Fabregat, J. 2010, A\&A, 509, A11

Meixner, M., Gordon, K. D., Indebetouw, R., et al. 2006, AJ, 132, 2268

Mokiem, M. R., de Koter, A., Vink, J. S., et al. 2007, A\& A, 473, 603

Negueruela, I., Steele, I. A., \& Bernabeu, G. 2004, Astronomische Nachrichten, 325, 749

Panagia, N. \& Felli, M. 1975, A\& $A, 39,1$

Porter, J. M. \& Rivinius, T. 2003, PASP, 115, 1153

Szczygiel, D. M., Stanek, K. Z., Bonanos, A. Z., et al. 2010, AJ, 140, 14

Vink, J. S., de Koter, A., \& Lamers, H. J. G. L. M. 2001, A\&\&A, 369, 574

Wisniewski, J. P. \& Bjorkman, K. S. 2006, ApJ, 652, 458

Wright, A. E. \& Barlow, M. J. 1975, MNRAS, 170, 41

\section{Discussion}

Miroshnichenko: What kind of positions do you have in your catalog of OB stars in the LMC (Spitzer, 2MASS, optical)?

Bonanos: We have used the best coordinates available, e.g. from Brian Skiff's updated lists for the Sanduleak catalog, which are generally accurate to $<1$ ".

WisNiEwski: How do you exclude or differentiate Herbig Be stars from classical Be stars in your data? Herbigs' transitional disks can show similar optical spectroscopic features: IR colors (especially given the dust content of the SMC/LMC) and candidate Herbigs have already been identified in the SMC/LMC, see e.g. Lamers et al. 1999; de Wit et al. 2002, 2003, 2005; Bjorkman et al. 2005.

Bonanos: We have not differentiated between them, as our sample was selected from the literature by mainly targeting OB stars in clusters. None of the stars in our catalog have HBe classifications, however some could be HBe stars. 